\title{
How long will social distancing take to work? Experts weigh in on Canada's COVID-19 response
}

\author{
n Cite as: CMAJ 2020 April 6;192:E382-3. doi: 10.1503/cmaj.1095857
}

Posted on cmajnews.com on March 20, 2020

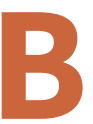

order closures, provinces declaring emergencies, scale-up of social distancing - Canada's response to coronavirus disease 2019 (COVID-19) rapidly escalated this week as case counts rose across the country. To put these developments in perspective, CMAJ reached out to infectious disease specialists Dr. Srinivas Murthy of BC Children's Hospital and the University of British Columbia in Vancouver, Dr. Matthew Oughton of the Jewish General Hospital and McGill University in Montreal, and Dr. Alon Vaisman of the University Health Network in Toronto.

\section{CMAJ: What do you make of the} changes in Canada's outbreak and response this week?

Oughton: This is the week when Canada began mitigation strategies in earnest. It is an unprecedented time for Canadians, as very few have ever before experienced such measures being invoked. The federal and provincial governments clearly recognize the need for a broad-based approach that includes economic stabilization as a critical part of mitigation; one cannot expect people to stay at home if they are concerned about paying the mortgage or needing groceries.

Murthy: Last week, the most notable thing was the cases of community transmission in Canada, and there's been an escalation of overall urgency attached to the outbreak response, which is warranted. As we're shifting from containment to mitigation, those concepts will have to be communicated very effectively by governments because that is going to be a very difficult transition for people to understand.

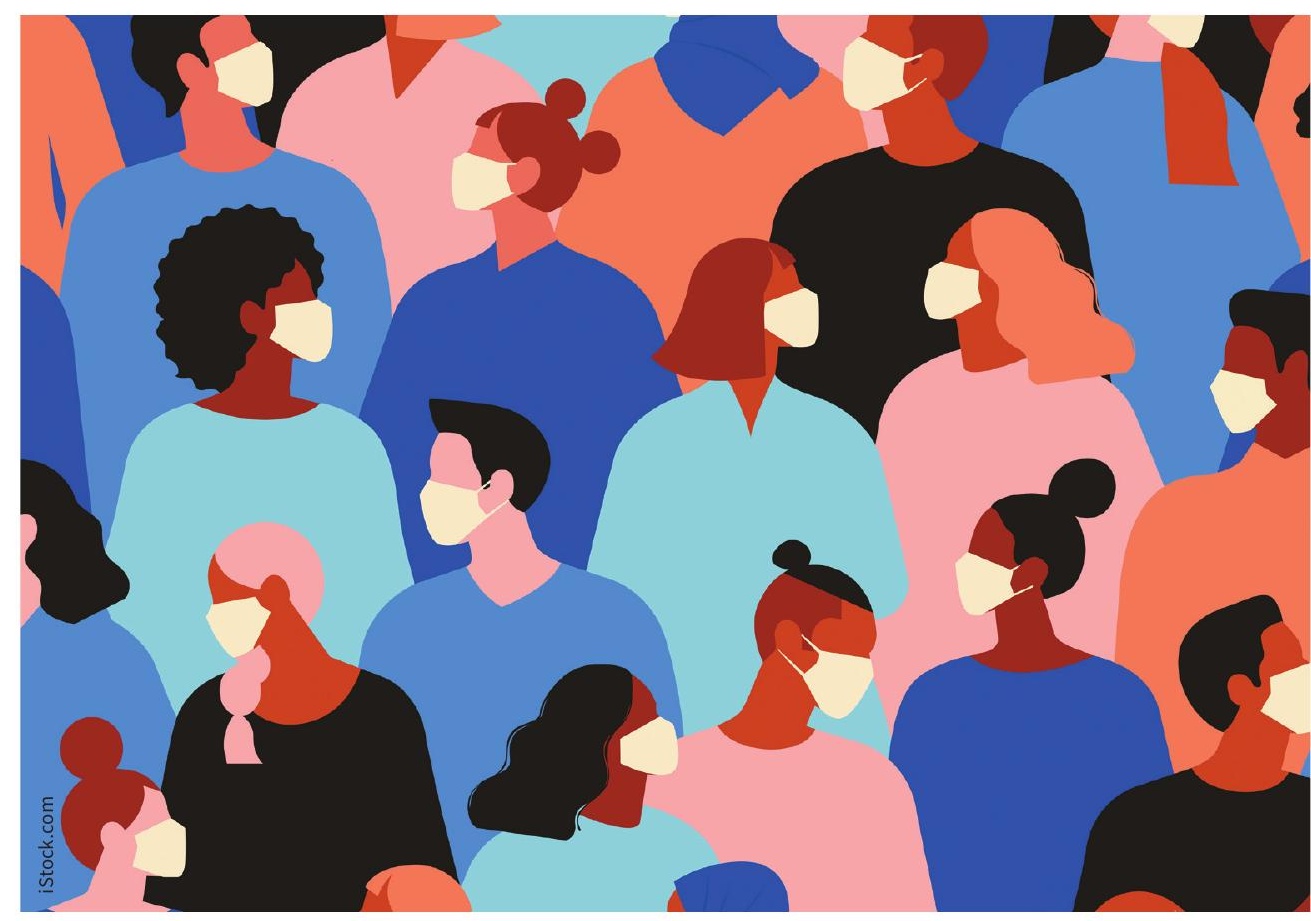

Canadians faced unprecedented restrictions on their social lives this week as governments stepped up mitigation efforts.

Vaisman: So far, social distancing efforts by the government have been well accepted by the public and widely supported by politicians and the media without much resistance. This is very reassuring to see.

\section{CMAJ: Given that we're now weeks} into the outbreak, why isn't Canada seeing more recovered cases?

Murthy: Most cases in Canada have [presented] in the past two or three weeks, and data from China and elsewhere show the usual recovery takes weeks and weeks, on the scale of a month. We've haven't seen [more recoveries] most likely because we haven't waited long enough.
Oughton: There is no indication that cases in Canada are requiring longer than usual to recover... Based on the diagnostic algorithms used during containment, testing was likely biased towards people with more severe symptoms, who would presumably require longer to recover, and people who had clinically mild disease may not have had follow-up evaluation to be classified as a recovered case. However, this question also illustrates an important point about how COVID-19 will affect our health care facilities. As an example, 14 days ago (March 5), Canada had 48 cases in total; as of this morning (March 20), 11 are recorded as recovered. This may indicate that COVID-19 patients require prolonged periods of hospitalization. 
Vaisman: The definition of recovery may change between jurisdictions... therefore, it may take a while to see [recoveries] confirmed by various public health agencies. The confirmation of recovery and reporting of these cases may be delayed due to burden on these agencies as well.

\section{CMAJ: How long might it take to see} the impact of social distancing? How will we know it's working?

Murthy: Daily new cases will be the metric that we'll follow to see if these things are working, and that likely won't happen for quite some time... maybe even months.

Oughton: [We'll need] a minimum of about two weeks to see effects from mitigation measures on new cases. This is due to the mean incubation period of [severe acute respiratory syndrome coronavirus 2] (approximately five to six days before first symptom) and the mean time required to develop symptoms severe enough that the patient will seek medical attention (approximately another six to seven days). Taken together, these time periods mean that positive tests today are reflecting transmission events that occurred about two weeks ago... It is very important for the public to understand this so that increasing numbers of cases during the first two weeks following mitigation measures are not seen as an indication that such measures are futile.
Vaisman: Because social distancing is such an inexact measure applied unevenly in various regions, it's hard to know what precise effect we should expect to see.

\section{CMAJ: A tweet circulated this week} showing a side-by-side comparison of Canada's infection rate versus Italy's. How do we compare? What can Canada learn from other countries this week?

Murthy: If you asked me that question a week ago, I would be much more optimistic about how we compared. Italy had 20 cases and then eight days later, they had 1200. [In contrast,] British Columbia had 20 cases and then eight days later they had 60 , so I was confident. But in the past few days, the case count and how it's rising across the country is making me a bit more anxious... A lot of people look to South Korea with its extensive testing, where they've done a couple hundred thousand tests, and Singapore with its very, very aggressive social distancing. Both have done a good job with the high escalation and then decline in their case counts over the past few weeks.

Vaisman: Canada's cases continue to rise at a rate that may mirror Italy's situation in the early phase of the outbreak. So far, we have yet to experience the massive burden on the health care system that Italy has experienced, and hopefully early adoption of social distancing will help prevent this... Early adoption is key. Even when case numbers appear low, once there is even a hint of community transmission, regions need to begin enforcing restrictions and social distancing.

\section{CMAJ: How do you expect the}

\section{situation in Canada to evolve in the} coming days?

Murthy: Our intensive care units, at least in British Columbia, are starting to feel the strain. We're not at Italy levels yet, but we're seeing more and more patients arrive... that's going to be the major conversation next week from a monitoring perspective, watching and making sure they have the resources they need.

Oughton: There will certainly be increases [in cases] across all provinces and territories at an accelerated rate until effects of mitigation strategies start to be reflected in the number of confirmed cases. I would also expect to see more measures to be announced by federal and provincial governments, including more economic supports for different industrial sectors, efforts to recruit manufacturers to produce more personal protective equipment, ventilators and other equipment needed for our hospitals and health care workers, and, very likely, stricter enforcement measures for people breaking quarantine.

\section{Lauren Vogel, CMAJ}

